\title{
Actitud hacia la Estadística en estudiantes de Odontología
}

\section{Artículo Original}

Clarisse Virginia Díaz-Reissner 1,a,b, María Elida Quintana-Molinas 2,a,c

${ }^{1}$ Universidad Nacional de Asunción. Facultad de Odontología. Dirección de Editorial y Estadística. Asunción, Paraguay.

${ }^{2}$ Universidad Nacional de Asunción. Facultad de Odontología. Cátedra de Salud Pública.

Asunción, Paraguay.

a Odontóloga

b Magíster en Estadística. Magíster en Metodología de la Investigación en Ciencias de la Salud

${ }^{c}$ Especialista en Endodoncia

\section{Attitude towards Statistics among students of Dentistry}

\section{Resumen}

Objetivo: Analizar la actitud hacia la estadística en estudiantes de grado de Odontología matriculados en la asignatura de Metodología de la Investigación y Bioestadística, en una universidad paraguaya, en el año 2015. Métodos: Se realizó un estudio cuasi-experimental. Se utilizó el cuestionario SATS-36 (Survey of Attitudes Toward Statistics) para medir la actitud hacia la estadística antes y después del curso. Resultados: La muestra quedó conformada por 43 estudiantes. El 83,72\% fue de sexo femenino, con una edad promedio de 19 $\pm 3,14$ años, el 48,96\% egresó del Bachillerato Humanístico y el 48,84\% deseaba obtener calificación "Muy Bueno". Basado en las puntuaciones obtenidas, presentaron un cambio de actitud negativa las dimensiones "dificultad" $(p=0,0176)$ y "esfuerzo" ( $p<0,0001)$, mientras que el cambio de actitud fue positivo para "competencia cognitiva" $(p=0,0470)$ y "valoración personal” $(p=0,0207)$. En la dimensión "cognitiva” el promedio fue significativamente mayor para los hombres $(p=0,035)$ y en la "afectiva" fue significativamente mayor para las mujeres $(p=0,019)$. La percepción acerca de la aptitud para las matemáticas disminuyó significativamente al finalizar el curso $(p=0,0001)$ El estrés generado por la Bioestadística fue medio en el $46,51 \%$. Conclusiones: Los estudiantes apreciaron el módulo de Bioestadística, consideraron una herramienta útil, tanto para uso personal como profesional. Con respecto a lo que creían inicialmente, la dificultad encontrada fue menor, por tanto se estresaron y esforzaron menos, pero consideraron que les faltaban aptitudes hacia las matemáticas. Por tanto, cuestionarios que miden la actitud podrían ser una herramienta útil para redireccionar estrategias docentes y promover una actitud positiva hacia la disciplina.

Palabras clave: Actitud; Estadística como asunto; Estudiantes de Odontología.

\section{Abstract}

Objective: To analyze the attitude towards statistics in undergraduate students of a Paraguayan university's Dentistry faculty enrolled in Investigation Methodology and Biostatistics' courses in 2015, was the objective of the investigation. Methods: A quasi-experimental study was conducted. The SATS-36 questionnaire (Survey of Attitude Toward Statistics) was used to measure the attitude toward statistics before and after the course. Results: The sample was conformed by 43 students, $83.72 \%$ was female, with 19 \pm 3.14 year-old age average, $48.96 \%$ graduated from the Humanistic Bachelor`s degree and $48.84 \%$ wanted to obtain a "Very Good" rating. Based on the scores obtained, the "difficulty" ( $p=0.0176)$ and "effort" $(p<0.0001)$ dimensions, showed a negative attitude change, while the positive attitude change was for "cognitive competence" $(p=0.0470)$

\section{Correspondencia:}

Clarisse Virginia Díaz Reissner

Correo electrónico: cdiazr@odo.una.py Universidad Nacional de Asunción. Facultad de Odontología. Avenida España c/Brasil. Asunción-Paraguay

Coautora:

María Elida Quintana-Molinas maria.e90una@hotmail.com

Conflicto de intereses: Los autores declaran no tener conflictos de interés.

Fuente de financiamiento: Autofinanciado

Fecha de recepción: 11/04/18

Fecha de aceptación: 22/05/18 
and "personal assessment" ( $p=0.0207)$. In the "cognitive" dimension the average was significantly higher for men $(p=0.035)$ and in the "affective" dimension it was significantly higher for women $(p=0.019)$. The perception for "aptitude for mathematics" decreased significantly at the end of the course $(p=0.0001)$ The stress generated by Biostatistics was medium in $46.51 \%$ of the sample. Conclusions: The students appreciated the Biostatistics module, considered it a useful tool, both for personal and professional use. With regard to their initially believed, the difficulty encountered was less, so their stress and effort were less, but they considered that they lacked aptitude for mathematics. Therefore, questionnaires that measure attitude could be a useful tool to redirect teaching strategies and promote a positive attitude towards the discipline.

Keywords: Attitude; Statistics as topic; Students dental.

\section{Introducción}

En el mundo actual, la estadística es de tanta importancia que en muchos países, esta forma parte del currículo de la educación secundaria e incluso se incluyen algunos tópicos en la educación primaria. El proceso de globalización y el desarrollo tecnológico hacen que la dinámica del mundo moderno exija que toda persona requiera de una cierta alfabetización en estadística ${ }^{1}$. Se debe tener en cuenta que una característica esencial de la estadística radica en que un mismo problema puede ser resuelto de diversas formas, pero se debe encontrar la manera más eficiente ${ }^{2}$.

La mayoría de los docentes se centra en qué contenidos enseñar y cómo enseñar, pero pasa por alto el hecho de que a muchos estudiantes no les gusta la disciplina, motivo por el cual estos sienten que no tienen la capacidad para comprender la estadística, creen que la misma no tiene ningún valor para la vida cotidiana y profesional, que es muy difícil de aprender o simplemente no están interesados en aprender, de esta manera no están dispuestos a esforzarse para aprender estadística y desarrollan una actitud negativa hacia la disciplina, sin considerar que las actitudes ejercen una gran influencia en la educación de la estadística ${ }^{3}$.

Resulta importante considerar la importancia de la actitud hacia la estadística, que debe ser abordada desde cursos introductorios, ya que estos podrían ejercer gran influencia en la conducta para abandonar o continuar con el curso y en optar por realizar cursos posteriores ${ }^{4}$.

Utilizar la estrategia de clase magistral como único método de enseñanza ya no resulta adecuado hoy día, teniendo en cuenta las múltiples posibilidades de métodos para la enseñanza-aprendizaje ${ }^{5}$. Se recomienda basarse en datos reales y utilizar ejemplos en cada área de estudio, de manera que esto les permita comprender la importancia de la estadística y crear un mayor interés, ya que pueden apreciar la utilidad en la profesión al interpretar los resultados ${ }^{6}$. Así también, el aprendizaje basado herramientas computacionales reduce la ansiedad y mejora la actitud hacia la estadística, mejorando el rendimiento ${ }^{7}$. Por otra parte, sugieren implementar cursos de nivelación de matemáticas básicas para estudiantes con menor aptitud numérica, así como también promover que los estudiantes reconozcan la aplicabilidad de la estadística no solo desde el enfoque de la investigación sino también que comprendan el conocimiento científico y su aplicabilidad ${ }^{8}$.

Por lo expuesto, el uso de las escalas de actitud hacia la estadística podrían ser de utilidad para el docente, ya que le ayudará identificar las actitudes negativas antes del proceso de enseñanza-aprendizaje, lo que le permitirá promover una mejor estrategia de desarrollo de clases durante el proceso de enseñanza, buscando de esta manera minimizar dichas actitudes ${ }^{9}$.

A lo largo de los años, han sido desarrolladas diversas escalas para medir la actitud hacia la estadística, se ha encontrado que existe una fuerte correlación entre las actitudes hacia la estadística, la ansiedad estadística y el desempeño en estadística. Las escalas que miden la actitud hacia la estadística son: Statistics Attitude Scale (Cruise, Cash, \& Bolton, 1985), Statistics Attitude Survey (Roberts \& Bilderback, 1980), Survey of Attitudes Toward Statistics (Schau, Stevens, Dauphinee \& Vecchio, 1995) y la Escala de Actitudes hacia la Estadística (Batanero, 2001). Todas estas escalas han resultados ser válidas y confiables internacionalmente ${ }^{10}$. Entre estas la escala SATS (Survey of Attitudes Toward Statistics) es una de las más utilizadas, la misma fue diseñada para identificar cuatro dimensiones de la actitud hacia la estadística, que son el "afectivo" que comprende los sentimientos positivos y negativos hacia la estadística, la "competencia cognitiva" que evalúa las actitud acerca de las habilidades intelectuales y conocimientos hacia la estadística, la "valoración" que evalúa actitudes acerca de la utilidad y la importancia de la estadísticas y la "dificultad" que abarca la actitud ante el manejo de la estadística de manera práctica ${ }^{5}$.

Por lo expuesto, para contribuir a la transformación de la enseñanza estadística, resulta relevante realizar estudios que pudieran mostrar y facilitar el interés de los docentes acerca de la dimensión de la actitud de los alumnos hacía la materia, de manera a evaluar las estrategias y actividades académicas que pudieran incidir en el cambio de actitud de manera favorable, fomentando mejoras en la manera de enseñar, no solo enfocándose en las destrezas y conocimientos, sino en la actitud generada, considerando que los alumnos pueden tener una experiencia de aprendizaje difícil. Se debe tener en cuenta que en la carrera de Odontología, generalmente se tiene un número reducido de horas de clase de Bioes- 
tadística dentro de otras asignaturas como Metodología de la Investigación o Salud Pública.

Por tanto, se planteó como objetivo del estudio analizar las actitudes hacia la estadística en estudiantes universitarios de grado de Odontología, matriculados en la asignatura de Metodología de la Investigación y Bioestadística.

\section{Métodos}

El diseño del estudio fue cuasi-experimental. La población de estudio lo conformaron estudiantes de grado de Odontología, de ambos sexos, que se encontraban matriculados en la asignatura de Metodología de la Investigación y Bioestadística, en una universidad paraguaya, en el ańo 2015. Se invitó a todos los alumnos a participar, completando la encuesta pre-test, el primer día de clases del módulo de Bioestadística. Al final del semestre se aplicó la encuesta pos-test, al finalizar el módulo de Bioestadística. Dicho módulo fue desarrollado en 40 horas reloj, en clases semanales de 4 horas, que abarcaron los siguientes contenidos: distribución de frecuencias, representación tabular y gráfica, medidas numéricas de resumen, distribución normal, métodos de muestreo, cálculo del tamaño de la muestra, interpretación de las pruebas de hipótesis, pruebas Chi-cuadrado y t de Student. Luego de cada contenido se desarrolló el análsis de artículos científicos acorde al tema abordado, de tal manera que pudieran aplicar los conocimientos adquiridos. Fueron excluidos del estudio los cuestionarios incompletos, sin la identificación correspondiente para los cuestionarios pre-test y/o pos-test y aquellos que no completaron ambos test. El protocolo de investigación fue evaluado y aprobado por el Comité de Ética de la institución, además se obtuvo la anuencia del docente encargado de la asignatura para su aplicación.

En cuanto a las estrategias de enseñanza-aprendizaje, se dictaron clases teóricas y se realizaron ejercicios prácticos, además se implementó la lectura e interpretación de artículos científicos con contenido estadístico.

Se utilizó el cuestionario de SATS-36 que fue provisto por la autora en la versión en inglés, bajo su autorización se sometió a un proceso de traducción, realizada por un médico y un odontólogo. Se realizó una prueba piloto donde se aplicó el cuestionario a 10 estudiantes a fin de detectar problemas de comprensión, no encontrándose necesario realizar ajustes del mismo. El formulario está compuesto por 36 ítems: seis referentes a la dimensión afectiva, seis a la competencia cognitiva, nueve a la valoración personal, siete para la dificultad, cuatro para el interés y cuatro para el esfuerzo. Los ítems del cuestionario pre-test y pos-test fueron idénticos, excepto que existieron pequeños cambios relacionados con la duración de la valoración, por lo que ambos aportaron medidas de actitudes hacia la materia en diferentes momentos del proceso de aprendizaje. Además, se incluyeron ítems adicionales (siete en el "pre" y seis en el "post") acerca de sus aptitudes y expectativas en cursos de matemática y estadística. Todos se evaluaron con una escala de Likert cuyas medidas van del $1 \mathrm{al} 7$, esto es, "muy en desacuerdo" a "totalmente de acuerdo". Siendo las afirmaciones tanto positivas como negativas, por lo que estás últimas tuvieron que ser invertidas para que toda la escala tuviera el mismo sentido. También se incluyeron características demográficas y académicas que forman parte del SATS-36. Se evaluó el cambio de actitud de dos maneras: a) basado en el rango intercuartil, se consideró como actitud neutra, tomando como referencia cuatro puntos, teniendo en cuenta una variación de: $1 / 2$ punto para las dimensiones afectivo, dificultad e interés; $3 / 4$ punto para la valoración y dos puntos para cognitivo y esfuerzo; y b) basado en la puntuación obtenida mediante la aplicación de estadística inferencial.

Para el análisis de datos se utilizó el programa InfoStat/L 2015. Se aplicó estadística descriptiva representada mediante frecuencia y porcentaje para las variables cualitativas, media y desviación estándar para variables cuantitativas. La estadística inferencial se utilizó para evaluar la diferencia en el cambio de la actitud hacia la estadística antes (pre-test) y después (pos-test) de cursar la materia mediante la prueba de Wilcoxon para muestras apareadas, y para comparar la diferencia de puntuación de cada dimensión por tipo de bachillerato de egreso se uitlizó la prueba U de Mann-Whitney y para comparar por sexo se utlizó la prueba $\mathrm{H}$ de Kruskal-Wallis, todos con un nivel de confianza del 95\%.

\section{Resultados}

Del total de 50 estudiantes, fueron excluidos siete debido a que abandonaron la carrera de Odontología. La muestra finalmente quedó conformada por 43 estudiantes. El $83,72 \%$ de la muestra fue de sexo femenino y el $16,28 \%$ de sexo masculino. La edad promedio fue de $19 \pm 3,14$ años, siendo la edad mínima de 17 y la máxima de 34 años. Aproximadamente la mitad de los estudiantes egresó del bachillerato humanístico y un 38,54\% considerado como otros, en diversos bachilleratos como admnistración, social, química y otros (Figura 1). Un poco menos de la mitad deseaba obtener una calificación de "muy bueno" en el módulo de Bioestadística (Figura 2).

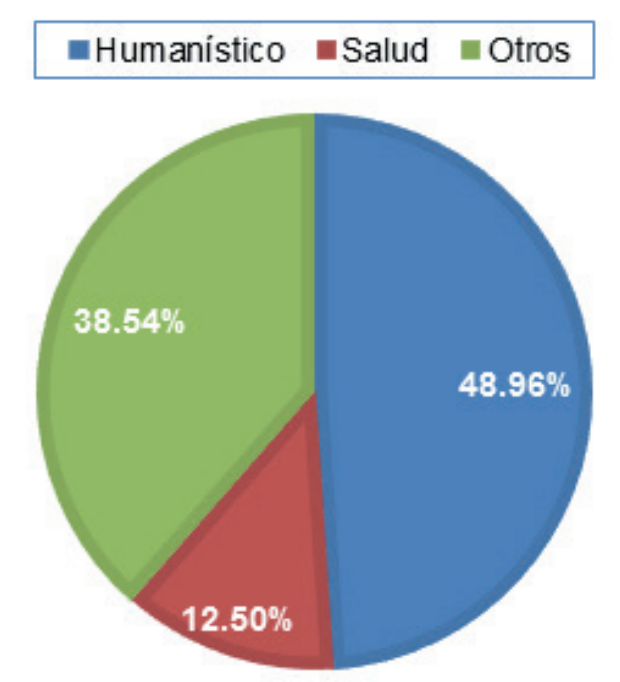

Figura 1. Bachillerato de egreso en estudiantes de Odontología. Paraguay, 2015 


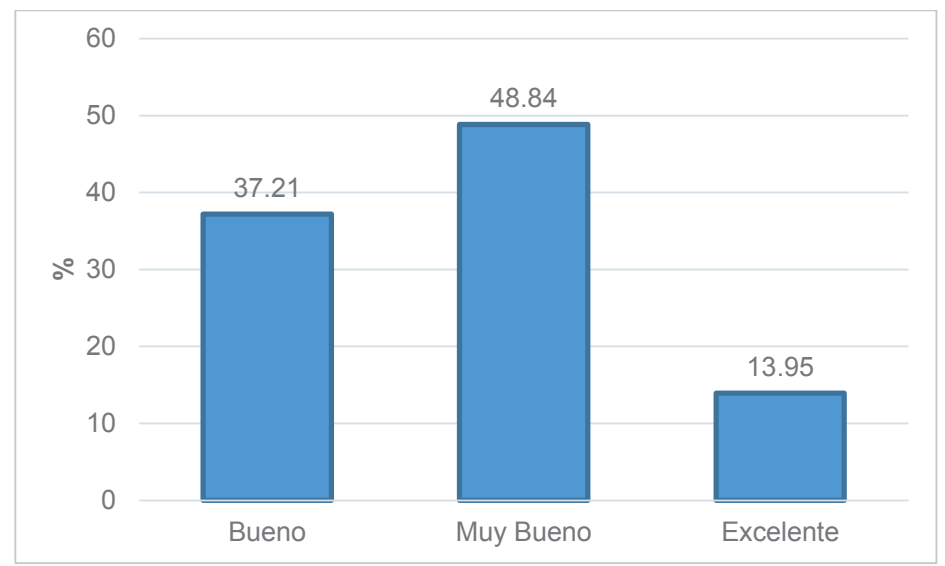

Figura 2. Calificación esperada en Bioestadística en estudiantes de Odontología. Paraguay, 2015

Ingresaron con una actitud neutra para las dimensiones: afectiva, cognitiva, dificultad y valoración; positiva: interés y esfuerzo. Se observó un cambio positivo solamente en valoracion, que pasó de neutro a positivo, sin embargo el interés y esfuerzo pasaron de postitivo a neutro (Tabla 1).

Tabla 1. Actitud hacia la estadística por dimensión en Bioestadística en estudiantes de Odontología. Paraguay, 2015

\begin{tabular}{lll}
\hline Dimensión & Pre-test & Pos-test \\
\hline Afectiva & Neutro & Neutro \\
Cognitiva & Neutro & Neutro \\
Valoración & Neutro & Positivo* \\
Dificultad & Neutro & Neutro \\
Interés & Positivo & Neutro* \\
Esfuerzo & Positivo & Neutro* \\
\hline
\end{tabular}

* Presentó cambio en la actitud, basado en el rango intercuartíl

Se observó que hubo una tendencia a disminuir, esto es, la opinión pasó a estar "muy en desacuerdo" con las afirmaciones positivas en las dimensiones dificultad, interés y esfuerzo; mientras que tuvieron una tendencia a aumentar las dimensiones afectiva, cognitiva y valoración personal (Figura 3). La dificultad encontrada también fue significativamente menor a la que creían inicialmente los estudiantes, igualmente el esfuerzo dedicado a la materia fue significativamente menor al finalizar el curso que lo declarado antes de conocer la materia, pero al contrario se vio aumentada significativamente la apreciación hacia la materia en sus dimensiones cognitiva y valoración personal. En la dimensión cognitiva los hombres presentaron un promedio significativamente más elevado que las mujeres, mientras que en la dimensión afectiva fueron las mujeres quienes presentaron el promedio significativamente más elevado. No se encontraron diferencias por tipo de bachillerato para ninguna de las dimensiones (Tabla 2).

En cuanto a las aptitudes y expectativas, se encontró una diferencia estadísticamente significativa en la percepción sobre aptitud para las matemáticas $(p=0,0001)$; la utilidad en estadística tampoco fue significativa $(p=0,9700)$ aunque tuvo una tendencia a ser positiva; no se encontró diferencia en cuanto al manejo de materiales estadísticos $(p=0,6265)$.

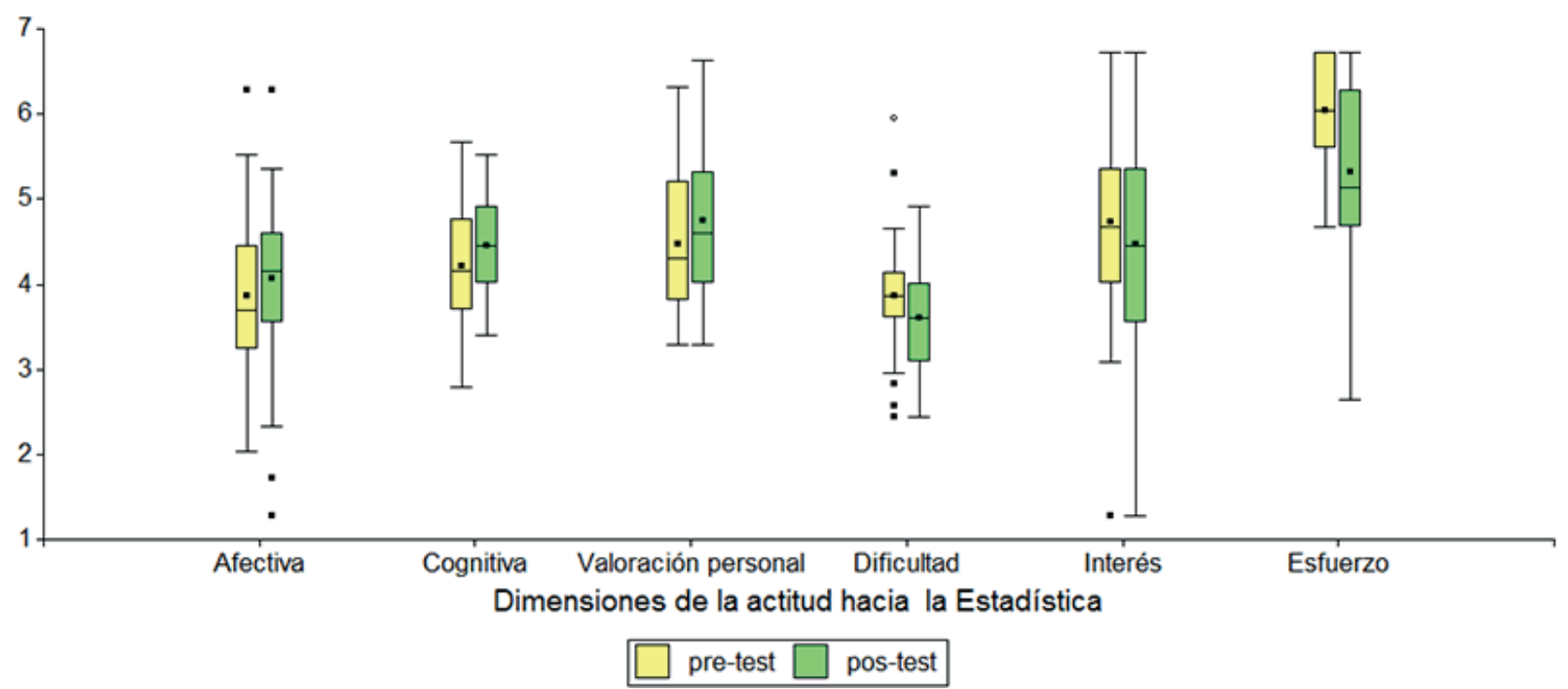

Figura 3. Actitud hacia la Estadística por dimensión en estudiantes de Odontología. Paraguay, 2015 
Tabla 2. Diferencia de actitud hacia la estadística por dimensión, comparada según sexo y bachillerato de egreso en Bioestadítica en estudiantes de Odontología. Paraguay, 2015

\begin{tabular}{lcccccc}
\hline Dimensión & Media (dif) & DE (dif) & $\mathbf{Z}$ & $\begin{array}{c}\text { Pos-pre test } \\
\text { Valor } \mathbf{p}\end{array}$ & $\begin{array}{c}\text { Sexo } \\
\text { Valor } \mathbf{p}\end{array}$ & $\begin{array}{c}\text { Bachillerato } \\
\text { Valor } \mathbf{p}\end{array}$ \\
\hline Afectiva & 0,23 & 1,21 & 1,46 & 0,1438 & $0,019^{*}$ & 0,920 \\
Cognitiva & 0,25 & 0,72 & 1,99 & $0,0470^{*}$ & $0,035^{*}$ & 0,319 \\
Valoración & 0,31 & 0,78 & 2,31 & $0,0207^{*}$ & 0,090 & 0,464 \\
Dificultad & $-0,30$ & 0,72 & $-2,37$ & $0,0176^{*}$ & 0,171 & 0,535 \\
Interés & $-0,28$ & 1,09 & $-1,43$ & 0,1531 & 0,714 & 0,748 \\
Esfuerzo & $-0,80$ & 1,05 & $-4,07$ & $<0,0001^{*}$ & 0,515 & 0,816 \\
\hline
\end{tabular}

*Estadísticamente significativo

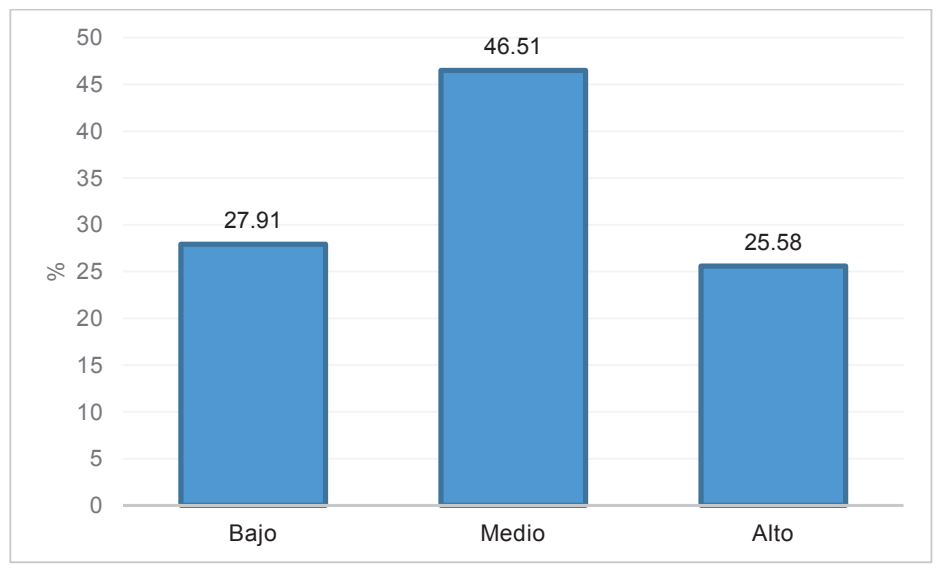

Figura 4. Nivel de estrés percibido al cursar el módulo de Bioestadística en estudiantes de Odontología. Paraguay, 2015

El 36,1\% obtuvo una calificación de aceptable, siendo las horas de estudio promedio 2,62 $\pm 1,64$. El nivel de estrés percibido fue medio en el $46,51 \%$ y alto en el 25,58\% (Figura 4).

\section{Discusión}

Los estudiantes de Odontología pasaron a valorar de manera neutra a positiva la estadística, aunque el interés y esfuerzo decreció de positivo a neutro. El análisis por puntuación arrojó mayores diferencias en las puntuaciones siendo más sensible al cambio, encontrándose que las dimensiones: cognitiva y valoración se incrementaron significativamente. Lo que podría atribuirse a la aplicación de una estrategia de enseñanza-aprendizaje que permitió ver la utilidad y aplicación en investigación, pero se debe tener en cuenta que también pudieron influir otros factores no considerados en el estudio. Con respecto a esto, en una revisión sistemática y meta-análisis encontraron que existe heterogeneidad entre los resultados obtenidos por dimensión refiriendo que entre otras cosas podría deberse al sexo y estructura del curso ${ }^{11}$. Otros autores sugieren que existe relación entre las características de los estudiantes; que a los hombres en general, les gusta más la estadística, creen tener buen conocimiento y habilidades para la disciplina, no se esfuerzan mucho por lo que, deben ser consideradas diversas características de los estudiantes ${ }^{12}$. En este estudio se encontró diferencias en la puntuación por sexo, coincidiendo en que los hombres presentaron un promedio significativamente más elevado para la dimension congnitiva. En cuanto la estructura del curso, la lectura de artículos científicos se consideró como una herramienta de aplicación al finalizar cada contenido temático, con lo que se esperaba aumentar el interés de la asignatura en los estudiantes, ya que se pretendía dar un panorama de la utilidad a lo largo de la carrera y de la vida profesional de la comprensión lectora de publicaciones científicas del área de Odontología.

En un estudio realizado en psicólogos, criminalistas y administradores que cursaban la carrera de grado se obtuvo como resultado que las dimensiones que variaron fueron la dificultad y la competencia cognitiva, ambas disminuyeron al final del curso ${ }^{13}$, coincidiendo el sentido del cambio con nuestro estudio para la dimensión dificultad, pero no coincidió para la competencia cognitiva debido a que aumentó al finalizar el curso.

Por otro lado, en un estudio realizado en un población de 2200 estudiantes matriculados en 101 secciones de post-secundaria en cursos de introducción a la estadística en todo Estados Unidos evaluaron los cambios en la actitud de los estudiantes al iniciar y finalizar el curso. Los resultados mostraron que en promedio los estudiantes ingresaron a los cursos con actitud neutra para las dimensiones afectiva y dificultad; positiva 
para cognitiva, valor e interés; y actitudes muy positivas para el esfuerzo. Sus actitudes aumentaron para afectivo, cognitiva y dificultad aunque no de manera significativa, pero disminuyeron para valor, interés y esfuerzo ${ }^{14}$. En este estudio, también se observó una actitud neutra para las mismas dimensiones pero además para la cognitiva, coincidiendo las otras dos dimensiones positivas e inclusive la muy positiva. Pero con respecto a la variación pos-test si hubo discrepancia, pues ninguna dimensión aumentó pero sí coincidió en la disminución del esfuerzo.

Con respecto a la capacidad para resolver problemas de matemáticas, en nuestro estudio al finalizar el curso, los estudiantes se sintieron con menos capacidad para resolver problemas de matemáticas que lo declarado al inicio, lo que podría deberse a diversos motivos. En un estudio realizado en estudiantes de grado de Medicina irlandeses encontraron que el sentimiento de los estudiantes acerca de su aptitud hacia las matemáticas en el pasado, podía predecir las actitudes futuras hacia la estadística, debido a que no diferenciaban entre las disciplinas de matemática y estadísica. Motivo por el cual, direccionar este problema de manera a reducir la ansiedad y la percepción de la dificultad podría ayudar a animar a los estudiantes a comprometerse con la estadística ${ }^{15}$. Otros resultados también evidenciaron que la estadística es abordada por los estudiantes con cierto prejuicio, variando sus actitudes en función de las experiencias previas ${ }^{16}$. Inclusive una mejor experiencia anterior en matemática o estadística tenían actitudes más positivas en los componentes: afectivo, cognitivo y dificultad. En otro estudio se encontró que después del curso de estadística aumentó significativamente el sentimiento negativo hacia las matemáticas, sintiéndose con menor capacidad que al iniciar un nuevo curso ${ }^{12}$.

Como limitación del estudio se puede mencionar que la muestra se extrajo solamente de una universidad, por lo que las generalizaciones de los resultados a otras instituciones de educación superior deben hacerse con precaución. El tamaño de la muestra también reduce la capacidad de generalizar los resultados. Repitiendo el estudio actual en instituciones similares donde se puedan ofrecer grupos de comparación homogéneos con diversas estrategias de enseñanza docente y la capacidad de generalizar los resultados a una población mayor que tenga validez externa permitirá optimizar el estudio.

Se concluye que tomar clases de estadística tuvo efectos tanto positivos como negativos sobre la opinión de los estudiantes, pues los estudiantes apreciaron el módulo de Bioestadística, lo consideraron una herramienta útil, tanto para uso personal como profesional. Con respecto a lo que creían inicialmente, la dificultad encontrada fue menor, por tanto se estresaron y esforzaron menos, pero consideraron que les faltaba aptitudes hacia las matemáticas. Es por esto, que la actitud hacia la estadística debería ser considerada por el docente, enfocándose en la estrategia de enseñanza-aprendizaje. Para su medición existen diversos instrumentos que permiten evaluar su eficacia. La escala SATS-36 evidenció ser una herramienta útil para evaluar la actitud hacia la estadística en estudiantes de Odontología, lo que podría permitir redireccionar la estrategia docente hacia una actitud positiva hacia la disciplina.

\section{Referencias bibliográficas}

1. León Salazar J. Enseñando estadística a futuros ingenieros. Sci Tech. 2007;XIII(34):563-8.

2. Zapata Cardona L. ¿Cómo contribuir a la alfabetización estadística? Rev Virtual Univ Católica Norte [Internet]. 2011[citado el 22 de mayo de 2018];(33). Disponible en: http://www.redalyc.org/articulo. oa?id= 194218961013

3. Schau C, Millar M, Petocz P. Research on Attitudes Towards Statistics. Stat Educ Res J. 2012;11(2):2-5.

4. Colon-Rosa H. Actiudes de estudiantes universitarios que tomaron cursos introductorios de estadísitca y su relación con el éxito académico en la disciplina [Tesis de doctorado]. [Puerto Rico]: Universidad de Puerto Rico; 2012. 205p.

5. Mutz R, Daniel H. University and student segmentation: Multilevel latent-class analysis of students' attitudes towards research methods and statistics. Br J Educ Psychol. 2013;83(2):280-304.

6. Bond M, Perkins S, Ramirez C. Students' Perceptions of Statistics: An Exploration of attitudes, conceptualizations, and content knowledge of statistics. Stat Educ Res J. 2012;11(2):6-25.

7. Ciftci S, Karadag E, Akdal P. Instruction of statistics via computer-based tools: Effects on statistics' anxiety, attitude, and achievement. J Educ Comput. Res. 2014;50(1):119-33.

8. Sesé A, Jiménez R, Montaño J, Palmer A. ¿Pueden las actitudes hacia la estadística y la ansiedad estadística explicar el rendimiento de los estudiantes? Revista de Psicodidáctica. 2015;20(2):285-304.

9. Medeiros C, de Paula J, Peres E. Evidências de validade da Escala Informatizada de Atitudes frente à Estatística - eSASPortuguês: um estudo correlacional. Psico-USF. 2011;16(3):357-65. http://doi.org/10.1590/S141382712011000300012

10. Escalante Gómez E, Repetto AM, Matinello G. Exploración y análisis de la actitud hacia la estadística en alumnos de psicología. Lib Rev Psicol. 2012;18(1):15-26.

11. Emmioğlu E, Capa-Aydin Y. Attitudes and achievement in Statistics: A Meta-Analysis Study. Stat Educ Res J. 2012;11(2):95-102.

12. Ramirez C, Schau C, Emmioglu E. The Importance of attitudes in statistics education. Stat Educ Res J. 2012;11(2):57-71.

13. Griffith J, Adams I, Gu L, Hart C, Nichols-Whitehead P. Students' attitudes toward statistics across the disciplines: A Mixed-Methods Approach. Stat Educ Res J. 2012;11(2):45-56.

14. Schau C, Emmioğlu E. Do introductory statistics sourses in the United States Improve students' attitudes? Stat Educ Res J. 2012;11(2):86-94. 
15. Hannigan A, Hegarty AC, McGrath D. Attitudes towards statistics of graduate entry medical students: the role of prior learning experiences. BMC Med Educ. 2014;14:70-70. http://doi.org/10.1186/1472-6920$14-70$

16. Ruiz de Miguel C. Actitudes hacia la estadística de los alumnos del grado en Pedagogía, Educación Social, y maestro de Educación Infantil y maestro de Educación Primaria de la UCM. Educacion XXI. 2015;18(2):35174. http://doi.org/10.5944/educXX1.12158 
